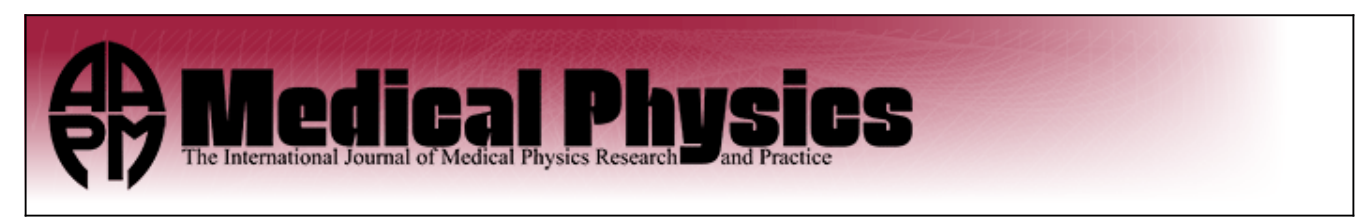

\title{
Introduction: Advances and trends in image formation in X-ray computed tomography
}

Frédéric Noo and Andrew Karellas

Citation: Medical Physics 42, 2656 (2015); doi: 10.1118/1.4918974

View online: http://dx.doi.org/10.1118/1.4918974

View Table of Contents: http://scitation.aip.org/content/aapm/journal/medphys/42/5?ver=pdfcov

Published by the American Association of Physicists in Medicine

\section{Articles you may be interested in}

Metal artifact correction for x-ray computed tomography using $\mathrm{kV}$ and selective $\mathrm{MV}$ imaging

Med. Phys. 41, 121910 (2014); 10.1118/1.4901551

Experimental comparison of grating- and propagation-based hard X-ray phase tomography of soft tissue

J. Appl. Phys. 116, 154903 (2014); 10.1063/1.4897225

Radial differential interior tomography and its image reconstruction with differentiated backprojection and projection onto convex sets

Med. Phys. 40, 091914 (2013); 10.1118/1.4812676

Multicontrast x-ray computed tomography imaging using Talbot-Lau interferometry without phase stepping Med. Phys. 39, 424 (2012); 10.1118/1.3672163

X-ray computed tomography: Advances in image formation

Med. Phys. 38, S1 (2011); 10.1118/1.3591339

"It can be reasonably postulated that verification of

the dose distribution inside

the patient was always the intent behind the secondary calculation process.."

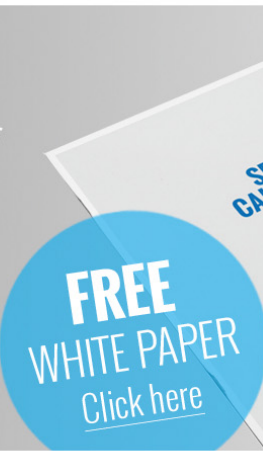

Free White Paper Secondary Calculations: Revisiting Rationale, Rethinking Methodology

NEW! DoseCHECK ${ }^{\text {TM }}$

3D Independent Secondary Dose Check

SUN NUCLEAR 


\title{
Introduction: Advances and trends in image formation in X-ray computed tomography
}

\author{
Frédéric $\mathrm{NoO}^{\mathrm{a})}$ \\ Utah Center for Advanced Imaging Research, University of Utah, Salt Lake City, Utah 84108 \\ Andrew Karellas \\ Department of Radiology, University of Massachusetts Medical School, Worcester, Massachusetts 01655
}

(Received 12 April 2015; accepted for publication 13 April 2015; published 4 May 2015)

[http://dx.doi.org/10.1118/1.4918974]

Research on image formation in x-ray computed tomography (CT) may currently be more active than it has ever been. New developments in data acquisition technology, and opportunities offered by advancing computer resources, are paving the way to further increase the role of CT. On the technology side, photon counting detectors that are suitable for body CT imaging are now emerging, and will undoubtedly lead $\mathrm{CT}$ to a new era, particularly as quantitative imaging based on x-ray attenuation of tissues could become more reliable. Such an advance will enable CT to become a major tool to monitor treatment of various diseases, particularly in oncology. Photon counting could also eradicate beam-hardening errors, preventing the need for additional imaging tests, such as MRI, after a CT scan.

Full utilization of the potential of photon counting detectors may require nonlinear image reconstruction techniques. Fortunately, advances in computer resources are enabling such techniques to become reality. Progress in nonlinear image reconstruction is moving at a fast pace right now, leveraging a wealth of novel optimization algorithms developed by mathematicians around the world. By enabling utilization of a more accurate model for the data acquisition procedure and properties of the image, nonlinear reconstruction methods have already been shown to be promising for dose reduction. Additional improvements are likely as additional physical effects can be modeled. Looking forward, the major thrust will be on continued efforts to reduce the radiation dose to patients by a combination of improved $\mathrm{x}$-ray detection techniques and model-based image reconstruction.

Clearly, $\mathrm{x}$-ray computed tomography is not limited anymore to diagnostic imaging. Cone-beam tomography using $\mathrm{x}$-ray transmission toward a flat panel detector has become an important component of treatment delivery and monitoring in oncology as well as in interventional radiology. Nonlinear reconstruction methods are now enabling a better handling of limited data geometries that are commonly encountered in such settings. Also, major progress is gradually being made on reducing artifacts due to physical effects such as scatter, beam hardening, and cone-beam geometry mishandling. These advances bring cone-beam CT imaging closer to the level of conventional fan beam CT quality. Recent advancements in detector and $\mathrm{x}$-ray tube technology are now also enabling dedicated $\mathrm{CT}$ of the breast for diagnostic imaging applications.

As progress is being made, it is important to keep in mind that robust image quality assessment is vital to success.
Task-based assessment of image quality using ROC analysis is one preferred approach. A significant research effort is needed to identify optimal ways of using ROC analysis for both the early-stage development and optimization of systems and the evaluation of adopted solutions. This need is well-understood with several research teams currently investigating these issues. Model observers for moderately complicated tasks are now available, as well as a number of tools for statistical analysis, which is integral to the utilization of ROC analysis.

In the "Third International Conference on Image Formation in X-Ray Computed Tomography" that was held in June 22-25, 2014 at Salt Lake City, UT, these and other issues were addressed with particular emphasis on the physical and mathematical aspects of image formation in CT. This meeting was attended by 154 imaging scientists and included 105 scientific presentations. The topics covered by these presentations included dose and image quality assessments, spectral CT imaging, model-based iterative reconstruction, cone-beam imaging in interventional radiology, and few view imaging.

All presentations were included in the conference proceedings, and the presenters were also given the opportunity to submit their presentation as a manuscript in this special focus series of Medical Physics. All manuscripts were peer reviewed according to the guidelines for all other publications in Medical Physics. The group of papers in this series represents the result of this process. The papers in this special series are published and archived as online only publications, which conveniently allows the utilization of color figures, but they are considered of equal value as other manuscripts that are published online and on paper. Additional information about the meeting, including the proceedings of all presented work can be found at: http://www.ucair.med.utah.edu/CTmeeting.

The attendees and presenters included some of the most prominent scientists in this field and there was also active participation by many of the new generation of CT scientists and students. This Third International Conference on Image Formation in X-Ray Computed Tomography was a great success and we are looking forward to the fourth meeting, which will take place in Bamberg, Germany, in 2016. We hope that the articles in this special focus series will provide important information that will accelerate progress in CT imaging.

\footnotetext{
a)Author to whom correspondence should be addressed. Electronic mail: noo@ucair.med.utah.edu
} 